\title{
Conditional Bistability, a Generic Cellular Mnemonic Mechanism for Robust and Flexible Working Memory Computations
}

\author{
Guillaume Rodriguez, ${ }^{1}$ Matthieu Sarazin, ${ }^{1}$ Alexandra Clemente, ${ }^{2,3}$ Stephanie Holden, ${ }^{2,3}$ Jeanne T. Paz, $, 2,4,5$ \\ and Bruno Delord ${ }^{1}$ \\ ${ }^{1}$ Institut des Systèmes Intelligents et de Robotique, Sorbonne Université, Centre National de la Recherche Scientifique, UMR 7222,75005 Paris, France, \\ ${ }^{2}$ Gladstone Institutes, San Francisco, California 94158, ${ }^{3}$ Neurosciences Graduate Program, ${ }^{4}$ Department of Neurology, University of California, \\ San Francisco, California, 94158, ${ }^{5}$ Kavli Institute for Fundamental Neuroscience, University of California San Francisco
}

Persistent neural activity, the substrate of working memory, is thought to emerge from synaptic reverberation within recurrent networks. However, reverberation models do not robustly explain the fundamental dynamics of persistent activity, including high-spiking irregularity, large intertrial variability, and state transitions. While cellular bistability may contribute to persistent activity, its rigidity appears incompatible with persistent activity labile characteristics. Here, we unravel in a cellular model a form of spike-mediated conditional bistability that is robust and generic. and provides a rich repertoire of mnemonic computations. Under asynchronous synaptic inputs of the awakened state, conditional bistability generates spiking/bursting episodes, accounting for the irregularity, variability, and state transitions characterizing persistent activity. This mechanism has likely been overlooked because of the subthreshold input it requires, and we predict how to assess it experimentally. Our results suggest a reexamination of the role of intrinsic properties in the collective network dynamics responsible for flexible working memory.

Key words: conditional bistability; flexible; generic; memory; robust; working memory

\section{Significance Statement}

This study unravels a novel form of intrinsic neuronal property: conditional bistability. We show that, thanks to its conditional character, conditional bistability favors the emergence of flexible and robust forms of persistent activity in PFC neural networks, in opposition to previously studied classical forms of absolute bistability. Specifically, we demonstrate for the first time that conditional bistability (1) is a generic biophysical spike-dependent mechanism of layer V pyramidal neurons in the PFC and that (2) it accounts for essential neurodynamical features for the organization and flexibility of PFC persistent activity (the large irregularity and intertrial variability of the discharge and its organization under discrete stable states), which remain unexplained in a robust fashion by current models.

\section{Introduction}

Working memory (WM), the ability to maintain and manipulate information within seconds, is essential to cardinal brain func-

Received July 13, 2017; revised Dec. 10, 2017; accepted Dec. 27, 2017.

Author contributions: A.C., S.H., J.T.P., and B.D. designed research; G.R., M.S., and B.D. performed research; G.R., M.S., and B.D. contributed unpublished reagents/analytic tools; G.R., M.S., and B.D. analyzed data; G.R., M.S., A.C., S.H., J.T.P., and B.D. wrote the paper.

This work was supported by the SMART Labex of the Agence Nationale de la Recherche (ANR; to G.R.), the Neuc 2016 program (Grant \#ANR-16-NEUC-0006-01) of the ANR and the Collaborative Research in Computational Neuroscience, and the National Science Foundation Graduate Research Fellowship (Grant \#1650113 to A.C.). We thank Jérémie Naudé for helpful discussions and comments on the manuscript.

The authors declare no competing financial interests.

Correspondence should be addressed to Bruno Delord, Institut des Systèmes Intelligents et de Robotique, Sorbonne Université, Centre National de la Recherche Scientifique, UMR 7222, 75005 Paris, France. E-mail: bruno.delord@upmc.fr. tions. Persistent neural activity represents a major neural correlate of WM, especially in the prefrontal cortex (PFC). The theory postulates that once triggered, persistent activity self-sustains through spiking reverberation in recurrent networks (Wang, 2001; Compte, 2006). Cortical architectures may provide sufficiently positive and nonlinear feedback for network dynamics to converge toward persistent activity (attractor dynamics; Cossart et al., 2003; MacLean et al., 2005). However, network reverberation as a unique causal origin remains controversial because it fails to robustly account for fundamental aspects of persistent activity such as the higher irregularity of spiking during the delay period of WM tasks, the large intertrial variability of 
the discharge and its temporal structure under quasi-stationary states, and the ability to encode parametric information (Seidemann et al., 1996; Koulakov et al., 2002; Compte et al., 2003; Goldman et al., 2003; Shafi et al., 2007; Barbieri and Brunel, 2008).

As a non-mutually exclusive alternative, intrinsic properties of neurons may underlie persistent activity, in interaction with synaptic mechanisms, for object (Compte, 2006), spatial (Camperi and Wang, 1998), and parametric (Koulakov et al., 2002; Goldman et al., 2003) WM, and the interaction of WM with long-term memory (Egorov et al., 2002; Larimer and Strowbridge, 2010). The intrinsic bistability of neurons (i.e., the coexistence of stable states of quiescence and self-sustained spiking) is central to this proposal because it allows memorizing transient inputs in individual neurons (Booth and Rinzel, 1995; Delord et al., 1996, 1997; Marder and Calabrese, 1996; Shouval and Gavornik, 2011). Bistability is ubiquitous in peripheral (Lee and Heckman, 1998; Perrier and Tresch, 2005), subcortical (Rekling and Feldman, 1997; Kawasaki et al., 1999), and cortical (Krnjević et al., 1971; Schwindt et al., 1988; Silva et al., 1991; Tahvildari et al., 2007; Zhang and Séguéla, 2010) structures, and in the PFC (HajDahmane and Andrade, 1997; Dembrow et al., 2010; Gee et al., 2012; Thuault et al., 2013).

This hypothesis has been criticized because intrinsic bistability is generally strongly stereotyped in vitro: it does not depend on the level of background depolarization [absolute bistability $(A B)$ ], requires long on- and off-stimuli (seconds), strong levels of pharmacological manipulations (e.g., neuromodulation), and displays extremely long (tens of seconds), high-frequency, highly regular discharges with partially inactivated spikes (Haj-Dahmane and Andrade, 1997; Egorov et al., 2002; Tahvildari et al., 2007; Zhang and Séguéla, 2010; Gee et al., 2012). These rigid features contrast with the flexibility of WM-related computational processes and persistent activity [e.g., high intertrial variability (Shafi et al., 2007) and irregular spiking (Compte et al., 2003)].

However, nonstereotype, conditional forms of bistability, where self-sustained spiking depends on background depolarization, have been found in the cortex (Silva et al., 1991; Tahvildari et al., 2007) and other structures (Bourque, 1986; Rekling and Feldman, 1997; Lee and Heckman, 1998; Kawasaki et al., 1999; Perrier and Tresch, 2005). Conditional bistability (CB) has been observed in layer V (L5) PFC pyramidal neurons (Thuault et al., 2013), which is not surprising, since bistability is underlain in these neurons by two spike-mediated (i.e., suprathreshold) currents - the high-threshold L-type calcium (CaL) and the calciumactivated nonspecific cationic (CAN) current (Haj-Dahmane and Andrade, 1997; Gee et al., 2012; Thuault et al., 2013) — that correlate with CB in many other neuronal types (Bourque, 1986; Silva et al., 1991; Rekling and Feldman, 1997; Lee and Heckman, 1998; Kawasaki et al., 1999; Perrier and Tresch, 2005; Tahvildari et al., 2007). A spike-mediated form of AB was previously studied (Shouval and Gavornik, 2011), but spike-mediated CB remains unexplored hitherto. Yet, its mechanism may depart from more classical spiking-independent forms of bistability relying on dendritic calcium (Hounsgaard and Kiehn, 1993; Booth and Rinzel, 1995), NMDA (Milojkovic et al., 2005; Major et al., 2008; Larimer and Strowbridge, 2010), or subthreshold currents (Delord et al., 1996, 1997; Washburn et al., 2000; Genet and Delord, 2002; Loewenstein et al., 2005; Carrillo-Reid et al., 2009; Genet et al., 2010).

Here, we explore the computational and mnemonic consequences of spike-dependent CB in a model of a L5 PFC pyramidal neurons.

\section{Materials and Methods}

Design of the standard model. We consider an isopotential L5 PFC pyramidal neuron model that follows the Hodgkin-Huxley formalism. The neuron model is endowed with the leak $\left(I_{L}\right)$ and action potential (AP) currents $\left(I_{N a}, I_{K}\right)$ and a synaptic ( $I_{S y n}$ " in vivo condition") or an injected ( $I_{I n j}$, "in vitro condition") input current. Depending on the hypothesis tested, the model also comprises one or more calcium- and/or voltagedependent suprathreshold currents, generically denoted $I_{\text {ion }}$. These currents can be depolarizing $\left(I_{C a L}, I_{C A N}\right)$ or hyperpolarizing [afterhyperpolarization potential potassium current $\left.\left(I_{A H P}\right)\right]$. The standard version of the model comprises the following three currents: $I_{\text {ion }}=I_{\mathrm{CaL}}+I_{\mathrm{CAN}}+$ $I_{\mathrm{AHP}}$, with parameters described in the Parameter section (see below). The membrane potential evolves according to the following:

$$
C \frac{d V}{d t}=-\left(I_{L}+I_{\mathrm{Na}}+I_{K}+I_{\text {ion }}+I_{\mathrm{Syn}}\right)+I_{\mathrm{Inj} \cdot}
$$

Leak current $I_{L}$ and action potential currents $I_{N a}$ and $I_{K}$. The leak current is written as follows:

$$
I_{L}=g_{L}\left(V-V_{L}\right),
$$

and AP currents are taken from a previous model we devised to reproduce spike currents of excitatory regular-spiking neocortical neurons (Naudé et al., 2012).

High-threshold calcium current $\left(I_{A H P}\right)$. The CaL current is derived from Delord et al. (1997) and follows as:

$$
I_{\mathrm{CaL}}=\bar{g}_{\mathrm{CaL}} x_{\mathrm{CaL}}^{2}\left(V-V_{\mathrm{CaL}}\right),
$$

where the activation $x_{\mathrm{CaL}}$ follows first-order kinetics:

$$
\frac{d x_{\mathrm{CaL}}}{d t}=\frac{x_{\mathrm{CaL}}^{\infty}(V)-x_{\mathrm{CaL}}}{\tau_{\mathrm{CaL}}(V)},
$$

with a voltage-dependent time constant:

$$
\tau_{\mathrm{CaL}}(V)=10^{\alpha_{\mathrm{CaL}}+\beta_{\mathrm{CaL}} V},
$$

with $\alpha_{C a l}$ and $\beta_{C a l}$ adapted to fit the time constant observed in vitro (Helton et al., 2005).

The activation follows:

$$
x_{\mathrm{CaL}}^{\infty}(V)=\left(1+\exp \frac{-\left(V-V_{1 / 2, \mathrm{CaL}}\right)}{K_{\mathrm{CaL}}}\right)^{-1},
$$

where $V_{1 / 2, \mathrm{CaL}}$ and $K_{\mathrm{CaL}}$, respectively, denote the half-activation potential and the e-fold slope of Boltzmann activation voltage dependence, and were estimated from the $I-V$ curve obtained in vitro (Helton et al., 2005).

Calcium-activated nonspecific cation current $\left(I_{\mathrm{CAN}}\right)$. The CAN current obeys the following:

$$
I_{\mathrm{CAN}}=\bar{g}_{\mathrm{CAN}} x_{\mathrm{CAN}}\left(V-V_{\mathrm{CAN}}\right),
$$

where the activation $x_{\mathrm{CAN}}$ follows first-order kinetics depending on the intracellular calcium concentration, as follows:

$$
\frac{d x_{\mathrm{CAN}}}{d t}=\frac{x_{\mathrm{CAN}}^{\infty}(\mathrm{Ca})-x_{\mathrm{CAN}}}{\tau_{\mathrm{CAN}}(\mathrm{Ca})},
$$

with

$$
\tau_{\mathrm{CAN}}(\mathrm{Ca})=\frac{1}{\alpha_{\mathrm{CAN}} C a+\beta_{\mathrm{CAN}}},
$$

and

$$
x_{\mathrm{CAN}}^{\infty}(\mathrm{Ca})=\frac{\alpha_{\mathrm{CAN}} \mathrm{Ca}}{\alpha_{\mathrm{CAN}} C a+\beta_{\mathrm{CAN}}},
$$

where $\alpha_{\mathrm{CAN}}$ and $\beta_{\mathrm{CAN}}$, respectively, denote activation and deactivation kinetic constants chosen to get significant activation in the micromolar range with time constants fitting those observed in vitro after large cal- 
cium influx in L5 PFC pyramidal neurons (i.e., $\sim 35 \mathrm{~ms}$ in the range 5-10 $\mu \mathrm{M}$ in the model and up to $\sim 100 \mathrm{~ms}$ at lower $\left[\mathrm{Ca}^{2+}\right]$ during interspike intervals (ISIs; Haj-Dahmane and Andrade, 1997)).

$\mathrm{I}_{A H P}$. The AHP current modeled here corresponds to the SK potassium channel type and obeys the following:

$$
I_{\mathrm{AHP}}=\bar{g}_{\mathrm{AHP}} x_{\mathrm{AHP}}^{2}\left(V-V_{\mathrm{AHP}}\right),
$$

where the activation $x_{\mathrm{AHP}}$ follows calcium-dependent first-order kinetics as follows:

$$
\frac{d x_{\mathrm{AHP}}}{d t}=\frac{x_{\mathrm{AHP}}^{\infty}(\mathrm{Ca})-x_{\mathrm{AHP}}}{\tau_{\mathrm{AHP}}(\mathrm{Ca})},
$$

with

$$
\tau_{\mathrm{AHP}}(\mathrm{Ca})=\frac{1}{\alpha_{\mathrm{AHP}} \mathrm{Ca}+\beta_{\mathrm{AHP}}},
$$

and

$$
x_{\mathrm{AHP}}^{\infty}(C a)=\frac{\alpha_{\mathrm{AHP}} \mathrm{Ca}}{\alpha_{\mathrm{AHP}} C a+\beta_{\mathrm{AHP}}},
$$

where $\alpha_{\mathrm{AHP}}$ and $\beta_{\mathrm{AHP}}$, respectively, denote the activation and deactivation kinetic constants, fitted to account for the time constants of medium AHPs observed in vitro in L5 PFC pyramidal neurons (Villalobos et al., 2004; Faber and Sah, 2007).

Calcium concentration dynamics. In the model, calcium concentration dynamics results from the inward influx due to $I_{\mathrm{CaL}}$ and from first-order buffering /extrusion (Haj-Dahmane and Andrade, 1997) as follows:

$$
\frac{d C a}{d t}=-\frac{1}{2 F} \frac{\text { Surf }}{\text { Vol }} I_{\mathrm{CaL}}+\frac{C a_{0}-C a}{\tau_{C a}},
$$

where $F$ is the Faraday constant, $C a_{0}$ is the basal intracellular calcium concentration, $\tau_{\mathrm{Ca}}$ is the buffering time constant, and the following:

$$
\frac{\text { Surf }}{\text { Vol }}=r_{1}^{-1}\left(1-\frac{r_{1}}{r_{0}}+\frac{r_{1}^{2}}{3 r_{0}^{2}}\right)^{-1},
$$

is the surface area-to-volume ratio of an idealized intracellular shell compartment of thickness $r_{1}$ situated beneath the surface of a spherical neuron soma of radius $r_{0}$. Calcium dynamics possesses an intrinsic asymmetry resulting from the inward influx due to rapid increases of $I_{\mathrm{CaL}}$ and the slower first-order buffering process.

Synaptic currents. In in vivo conditions (see Protocols), synaptic activity is simulated with fluctuating excitatory AMPA and inhibitory $\mathrm{GABA}_{\mathrm{A}}$ conductances as studied in L5 PFC pyramidal neurons (Destexhe and Paré, 1999), and the synaptic current is modeled as follows:

$$
I_{\text {Syn }}=g_{E}\left(V-V_{E}\right)+g_{1}\left(V-V_{1}\right),
$$

where $V_{E}$ and $V_{I}$ are the reversal potentials, and the fluctuating conductances $g_{E}$ and $g_{I}$ are given by two Uhlenbeck-Ornstein processes, as follows:

$$
\begin{gathered}
\frac{d g_{E}}{d t}=\left(g_{E}-g_{E_{0}}\right) / \tau_{E}+\sigma_{E} x_{E}(t), \\
\frac{d g_{I}}{d t}=\left(g_{I}-g_{I_{0}}\right) / \tau_{I}+\sigma_{I} x_{I}(t),
\end{gathered}
$$

where $\tau_{E}$ and $\tau_{I}$ are the respective time constants of the temporal evolution of conductances, $g_{E 0}$ and $g_{I 0}$ are the mean conductances (that depend on the considered protocol), $\sigma_{E}$ and $\sigma_{I}$ are the SDs, and $x_{E}(t)$ and $x_{I}(t)$ are Gaussian stochastic processes with zero mean and unit SDs.

Determination of afterdepolarization potential amplitudes. The amplitude of afterdepolarization potentials (ADPs) is determined using a specific stimulation protocol composed of a $15 \mathrm{~ms}$ phasic current of fixed amplitude set to elicit a single action potential. The ADP amplitude is calculated as the maximal membrane potential difference between con- ditions in the presence and the absence of the tested supraliminar current $\left(I_{\mathrm{CaL}}, I_{\mathrm{CAN}}\right.$, or both). This difference was calculated in a window starting 10 $\mathrm{ms}$ after the action potential peak (to avoid the influence of different action potential lengths due to the presence/absence of suprathreshold currents) and ending $1 \mathrm{~s}$ later, far after complete relaxation to resting potential.

Standard model parameters. Unless indicated in figure legends, standard parameter values are as follows: for the leak current, $g_{L}=0.05 \mathrm{mS}$. $\mathrm{cm}^{-2}, V_{L}=-70 \mathrm{mV}$; AP current parameters are as in a previous model that we developed of excitatory regular-spiking neocortical neurons (Naudé et al., 2012), with $\bar{g}_{\mathrm{Na}}=24 \mathrm{mS} \cdot \mathrm{cm}^{-2}, V_{\mathrm{Na}}=50 \mathrm{mV}, \bar{g}_{K}=3 \mathrm{mS} \cdot \mathrm{cm}^{-2}$, and $V_{K}=-90 \mathrm{mV}$. For supraliminar ionic currents, parameters are $\bar{g}_{\mathrm{CaL}}=$ $0.0045 \mathrm{mS} \cdot \mathrm{cm}^{-2}, V_{\mathrm{CaL}}=150 \mathrm{mV}, V_{1 / 2, \mathrm{CaL}}=-12 \mathrm{mV}, K_{\mathrm{CaL}}=7 \mathrm{mV}$, $\alpha_{\mathrm{CaL}}=0.6, \beta_{\mathrm{CaL}}=-0.02 \mathrm{mV}^{-1}, \bar{g}_{\mathrm{CAN}}=0.025 \mathrm{mS} \cdot \mathrm{cm}^{-2}, V_{\mathrm{CAN}}=30$ $\mathrm{mV}, \alpha_{\mathrm{CAN}}=0.0056 \mu \mathrm{M}^{-1} \cdot \mathrm{ms}^{-1}$ and $\beta_{\mathrm{CAN}}=0.0125 \mathrm{~ms}^{-1}$, $\bar{g}_{\mathrm{AHP}}=0.2 \mathrm{mS} \cdot \mathrm{cm}^{-2}, V_{\mathrm{AHP}}=-90 \mathrm{mV}, \alpha_{\mathrm{AHP}}=0.05 \mu \mathrm{M}^{-1} \cdot \mathrm{ms}^{-1}$, and $\beta_{\mathrm{AHP}}=0.2 \mathrm{~ms}^{-1}$. Geometrical and intracellular calcium dynamics parameters are as follows: $F=96,500 \mathrm{~mol} \cdot \mathrm{s}^{-1} \cdot A^{-1}, r_{0}=4 \mu \mathrm{m}, \mathrm{r}_{1}=$ $0.25 \mu \mathrm{m}, \mathrm{Ca}_{0}=0.1 \mu \mathrm{M}, \tau_{\mathrm{Ca}}=100 \mathrm{~ms}$. Synaptic parameters are $g_{\mathrm{E}_{0}, \mathrm{BACKGROUND}}=0.0325 \mathrm{mS} \cdot \mathrm{cm}^{-2}, g_{E_{0}, \text { EVENT }}=0.065 \mathrm{mS} \cdot \mathrm{cm}^{-2}$, $g_{E_{0}, \text { DELAY }}=0.040 \mathrm{mS} \cdot \mathrm{cm}^{-2}$, and independently of the period considered, $\sigma_{E}=0.0125 \mathrm{mS} \cdot \mathrm{cm}^{-2}, g_{I_{0}}=0.1 \mathrm{mS} \cdot \mathrm{cm}^{-2}, \sigma_{I}=0.0075 \mathrm{mS} \cdot \mathrm{cm}^{-2}, \tau_{\mathrm{E}}$ $=2.5 \mathrm{~ms}, \tau_{\mathrm{I}}=10 \mathrm{~ms}, V_{E}=0 \mathrm{mV}$, and $V_{I}=-75 \mathrm{mV}$.

Numerical procedures. The models were numerically integrated using the forward Euler method with a $1 e^{-2}$ ms time step. Bifurcation diagrams were obtained using the XPP software for qualitative analysis of dynamical systems (http://www.math.pitt.edu/ bard/xpp/xpp.html). Spikes were detected as a maximum of the membrane potential above $-20 \mathrm{mV}$.

In in vitro protocols, the behavior maps (see Figs. 2, 3) were built as follows: the discharge during a 10 s delay period was classified as (1) memoryless, when no spike occurred during the delay period or when one spike occurred at $<25 \mathrm{~ms}$ after the onset of the delay period; (2) transient memory, when an unstable discharge occurred during the delay period and lasted at least $25 \mathrm{~ms}$ after the delay period onset (to exclude cases where an ultimate spike is blown just after the phasic current pulse due to the activation of a fast sodium current in the last milliseconds of the phasic current pulse); and (3) stable memory, when the last spike of the discharge occurred after $9.5 \mathrm{~s}$ and the mean relative absolute difference between successive ISIs was $<5 \%$ during the last $2 \mathrm{~s}$ of the delay period.

In in vivo protocols, spikes were defined as belonging to a burst when they were part of a succession of at least three spikes with all ISIs $<100 \mathrm{~ms}$ (instantaneous frequencies $>10 \mathrm{~Hz}$ ). Other spikes were defined as not belonging to a burst [i.e., isolated spikes or doublet of spikes (with an intradoublet ISI inferior to $100 \mathrm{~ms}$ ) that were separated from the rest of the spike train by ISIs $>100 \mathrm{~ms}$ ]. Bursting episodes were defined as contiguous periods of time within which all spikes belonged to a burst. Nonbursting episodes were defined as the periods outside the bursting epidsodes. The choice of $100 \mathrm{~ms}$ as a cutoff ISI value was arbitrarily set to separate periods with frequency inferior to $10 \mathrm{~Hz}$, which are typical of the spontaneous state of activity in the awake cortex from periods of activity taking part in coding (Destexhe et al., 2001). This exact value is not important to the conclusions drawn in the present study.

Statistical methods. We used a two-tailed Wilcoxon rank-sum test to compare the medians of the $\mathrm{CV}$ distributions in the event and delay periods of the event/delay protocol, because the CV distributions were not normal, according to Kolmogorov-Smirnov goodness-of-fit hypothesis tests. A similar procedure was used to compare the medians of the $\mathrm{CV}_{2}$ distributions in the event and delay periods of the event/delay protocol.

\section{Results}

\section{Mimicking synaptic inputs during WM}

Our main goal was to determine whether depolarizing spikemediated currents can maintain the memory of an event at the level of the discharge of an individual neuron, while producing realistic spiking patterns, as observed during WM. To that end, we designed a realistic isopotential model of a L5 pyramidal PFC neuron endowed with high-threshold $\mathrm{CaL}\left(I_{\mathrm{CaL}}\right)$, calcium-activated nonspecific cationic $\left(I_{\mathrm{CAN}}\right)$, afterhyperpolarization potassium $\left(I_{\mathrm{AHP}}\right)$, 
event protocol
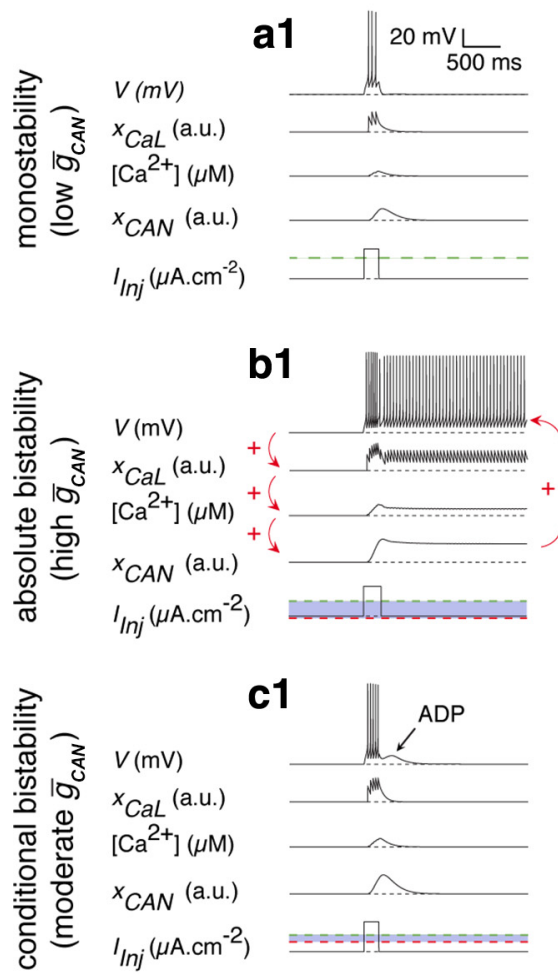

event/delay protocol

bifurcation diagrams
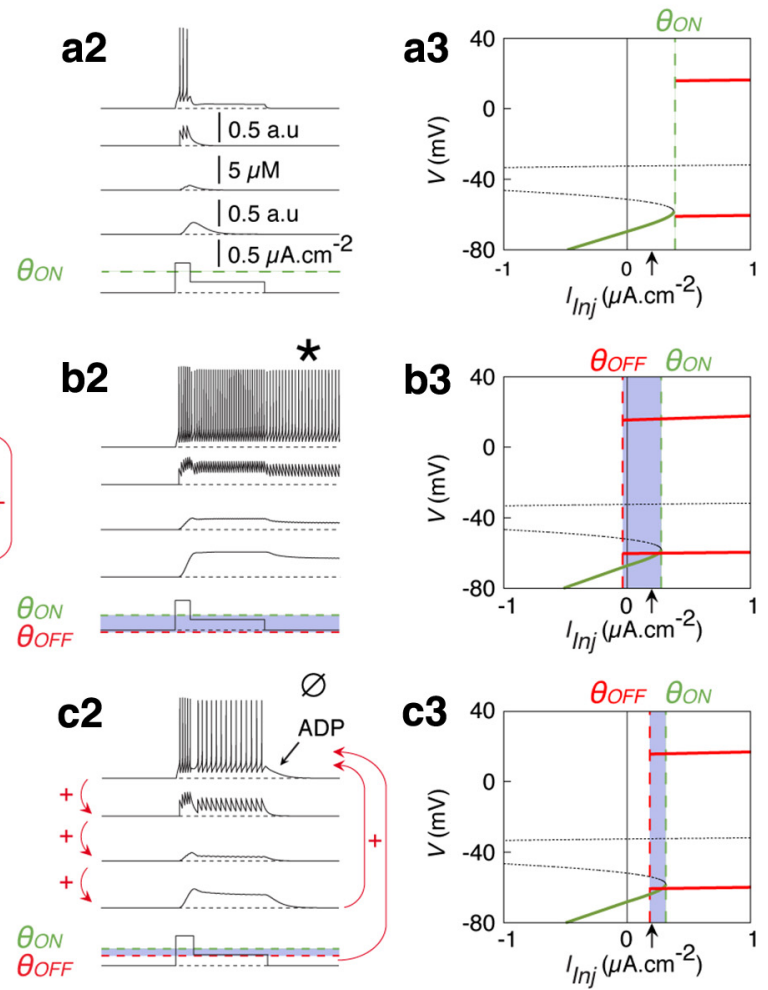

Figure 1. Conditional bistability is a hidden property in neurons endowed with a suprathreshold conductance in response to standard protocols applied in vitro. a1-c3, The response of monostable $\left(\boldsymbol{a} 1-\boldsymbol{a} 3 ; g_{C A N}=0.003 \mathrm{~ms} \cdot \mathrm{cm}^{-2}\right)$, absolute bistable $\left(\boldsymbol{b} \mathbf{1}-\boldsymbol{b} 3 ; g_{C A N}=0.03 \mathrm{~ms} \cdot \mathrm{cm}^{-2}\right)$, and conditional bistable $\left(\boldsymbol{c} \mathbf{1}-\mathbf{c} 3 ; g_{C A N}=0.02 \mathrm{~ms} \cdot \mathrm{cm}^{-2}\right)$ neurons $($ standard model $)$ to an event protocol with a $0.2 \mathrm{~s}$ suprathreshold current $\operatorname{step}(\boldsymbol{a 1}, \boldsymbol{b 1}, \mathbf{c} \mathbf{1})$ and to the event-delay protocol, in which the event is followed by a $1 \mathrm{~s} \mathrm{subthreshold} \mathrm{depolarizing} \mathrm{current} \mathrm{mimicking} \mathrm{background}$ activity in PFC networks during the delay of a working memory task $(\boldsymbol{a} \mathbf{2}, \boldsymbol{b} \mathbf{2}, \boldsymbol{c} \mathbf{2})$. Note that the discharge is continuing after the delay stimulus in the absolute bistable neuron $(\boldsymbol{b} \mathbf{2}$, star symbol). Note the $\operatorname{ADP}\left(\mathbf{c} 2\right.$, void symbol) following spiking in the conditional bistable neuron. The thresholds for initiating $\left(\theta_{O N}\right)$ and terminating $\left(\theta_{O F F}\right)$ spiking are represented as green and red dotted lines,

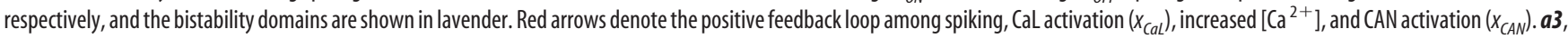
$\boldsymbol{b} 3, \mathbf{c} 3$, Right panels, Bifurcation diagrams illustrating the stable fixed point (resting potential, green solid curve) and the minimal/maximal potentials of action potentials during the limit cycle (rhythmic spiking, red solid curves), the thresholds for initiating $\left(\theta_{O N^{\prime}}\right.$ green doted lines) and terminating spiking $\left(\theta_{\text {OFF, }}\right.$ red doted lines), and the $I_{\text {Inj-delay }}$ during the delay (black arrows). Bistability domains are shown in lavender. Black dotted lines indicate unstable fixed points of the models.

action potential and leak currents, and intracellular calcium ( $\left[\mathrm{Ca}^{2+}\right]$ ) linear dynamics (see Materials and Methods). In the model, $I_{\mathrm{CaL}}$ and $I_{\mathrm{CAN}}$ are spike mediated because $I_{\mathrm{CaL}}$ activates at membrane potentials above the spike threshold and is the unique source of intracellular calcium activating $I_{\mathrm{CAN}}$, as found in PFC neurons exhibiting spike-mediated bistability (Haj-Dahmane and Andrade, 1997). Parameters were set such that $I_{\text {CAN }}$ was the sole spikemediated charge carrier between these two currents (Haj-Dahmane and Andrade, 1997).

To test whether spike-mediated currents contribute to persistent activity in the model, we used two stimulation protocols. The event protocol, classically used to assess bistability, consisted of a single, short (0.2 s) suprathreshold current pulse mimicking the arrival of an input (e.g., perceptive or motor) event. In the event/ delay protocol, the event was followed by a longer (1 s) subthreshold depolarizing current mimicking background activity from the PFC network to the neuron during the delay of a WM task. This input may correspond to persistent activity reverberating within local PFC recurrent connections to maintain information about the event or to ongoing inputs related to motivational, attentional, anticipatory, or executive aspects of WM processes.

\section{Conditional bistability is invisible with classical protocols}

At low levels of the maximal CAN conductance $\left(g_{\mathrm{CAN}}\right)$, the neuron discharged only during the event (Fig. 1a1, event protocol), even when the event was followed by a background subthreshold delay current (Fig. 1a2, event/delay protocol). A bifurcation analysis as a function of the $I_{\text {Inj }}$ indicated that the neuron was monostable (M): it admitted either a stable fixed point corresponding to the resting potential (Fig. 1a3, green solid curve) or, above the spiking threshold $\theta_{\mathrm{ON}}$, to a stable limit cycle corresponding to rhythmic spiking (Fig. 1a3, red solid curves).

At large $g_{C A N}$ values, the event induced a self-sustained discharge that outlasted the triggering event, providing a cellular form of memory, with both protocols (Fig. 1b1,b2). Mechanistically, self-sustained spiking arose from the positive feedback among spiking, $\mathrm{CaL}$ activation, increased $\left[\mathrm{Ca}^{2+}\right]$, and CAN activation (Fig. 1b1, red arrows), which did not operate at low $g_{\mathrm{CAN}}$ levels (compare with Fig. 1a1). Here, the neuron was bistable: the resting potential coexisted with rhythmic spiking in a bistability domain situated between $\theta_{\mathrm{ON}}$, the threshold for initiating spiking, and $\theta_{\mathrm{OFF}}$, the threshold for terminating spiking (Fig. 1b3, lavender domain). The bistability domain included $I_{\text {Inj }}=0 \mu \mathrm{A}$. $\mathrm{cm}^{-2}\left(\theta_{\mathrm{OFF}}<0<\theta_{\mathrm{ON}}\right)$, so that cellular memory did not require any background subthreshold input. Hence, the spike-mediated bistability was absolute, as observed in a previous model (Shouval and Gavornik, 2011) and in PFC neurons under pharmacological manipulations (Dembrow et al., 2010; Gee et al., 2012). Therefore, persistent activity outlasted the delay period (Fig. 1b2, star; 


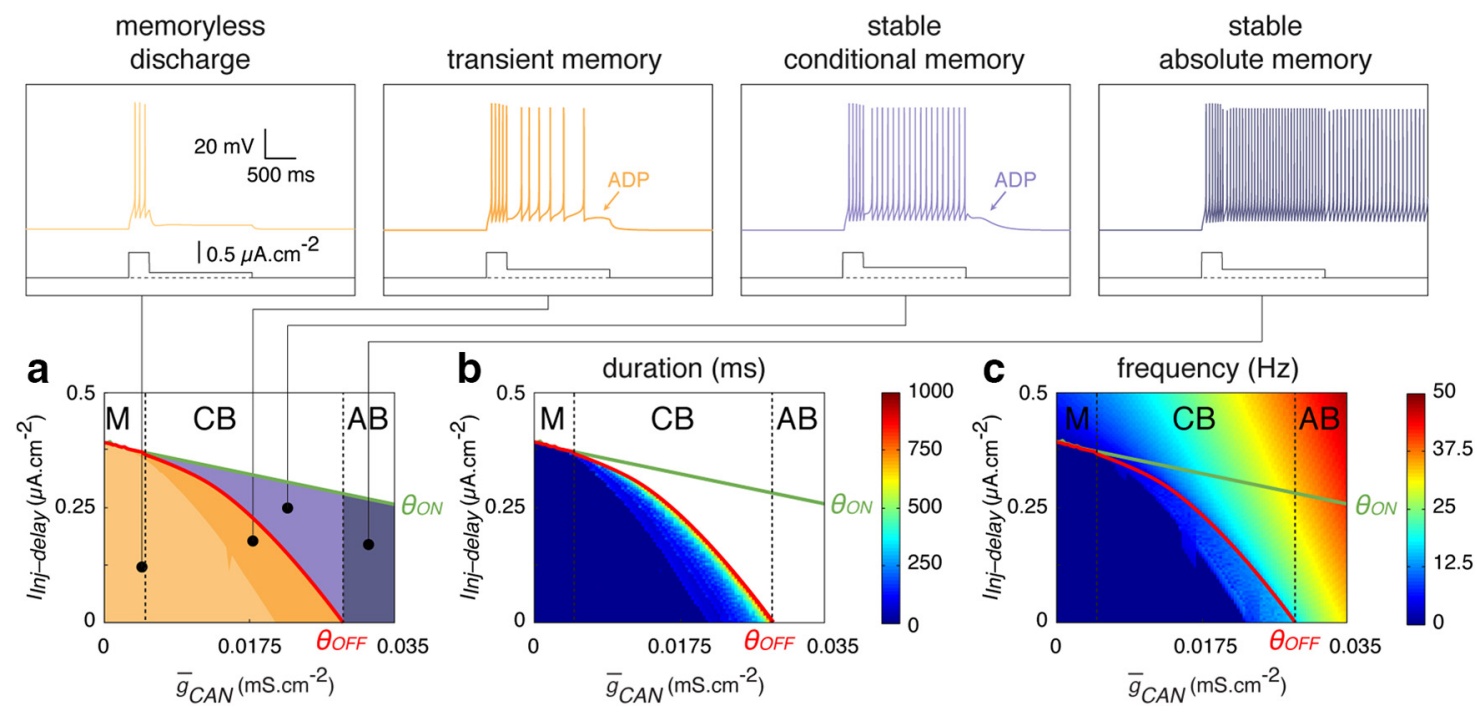

Figure 2. Existence and expression of conditional bistability in vitro. $\boldsymbol{a}$, Discharge behaviors of the standard neuron model in response to the event/delay protocol $\left(I_{\operatorname{lnj} \text {-event }}=0.6 \mu \mathrm{A} \cdot \mathrm{cm}^{-2}\right)$ as a function of the $g_{C A N}$ and the $I_{\text {Inj-delay }} \cdot M, C B$, and $A B$ indicate the monostability, conditional bistability, and absolute bistability domains, respectively. The memoryless and transient memory, stable conditional memory and stable absolute memory behaviors are indicated, respectively, as yellow, orange, lavender, and purple domains (bottom) and discharges (top panels). $\boldsymbol{b}, \boldsymbol{c}$, Heat maps of the duration of memoryless and transient memory behaviors $(\boldsymbol{b})$ and of the mean firing frequency of stable memory behaviors (c) during the delay period, as a function of the $g_{C A N}$ and the $I_{I n j-d e l a y}$. Note that above $\theta_{O N}$, the tonic current is suprathreshold and the neuron fires even when no event precedes the delay period. Sawteeth at the border between memoryless and transient regions correspond to the discharge of one spike occurring at $>25 \mathrm{~ms}$ after the onset of the delay period, while the main part of the transient memory region corresponds to the discharge of several spikes during the delay period (see definition of transient memory behavior in Materials and Methods).

i.e., memory was infinite), unless a specific inhibitory input terminated it.

At intermediate $g_{\text {CAN }}$ levels, we observed that bistability was conditional: spiking during the delay depended on the level of subthreshold depolarization, as found in several neural structures and in the PFC (Bourque, 1986; Silva et al., 1991; Rekling and Feldman, 1997; Lee and Heckman, 1998; Kawasaki et al., 1999; Perrier and Tresch, 2005; Tahvildari et al., 2007; Thuault et al., 2013). After the event, spiking stopped in the event protocol (Fig. 1c1) but persisted during the entire delay in the event/delay protocol (Fig. 1c2) even though the background delay current $\left(I_{\text {Inj-delay }}\right)$ was subthreshold (i.e., below $\theta_{\mathrm{ON}}$; Fig. $1 c 3$, black arrow). This was possible because $I_{\text {Inj-delay }}$ was above $\theta_{\text {OFF }}$ (i.e., in the bistability domain; $0<\theta_{\mathrm{OFF}}<\theta_{\mathrm{ON}}$; Fig. 1c3). The background current was needed under $\mathrm{CB}$, by contrast to $\mathrm{AB}$, because the spike-mediated positive feedback was not sufficient to support autonomous self-sustained spiking at moderate $g_{C A N}$ levels. This explains why persistent activity terminated at the end of the delay when $I_{\text {Inj }}$ values returned to zero, below $\theta_{\mathrm{OFF}}$ and the bistability domain (Fig. 1c2, void symbol), being followed by an ADP (Fig. 1c2, black arrow), as found in PFC neurons expressing spike-mediated currents and/or bistability (Haj-Dahmane and Andrade, 1997; Dembrow et al., 2010; Gee et al., 2012). Thus, under $\mathrm{CB}$, the duration of cellular memory adapted to the duration of network memory (i.e., reverberation), alleviating the requirement for a dedicated inhibitory stimulus to terminate persistent activity. Note also that triggering spiking-dependent bistability did not require long stimulations, because of the moderate time constant of $I_{\mathrm{CAN}}(\sim 100 \mathrm{~ms}$; see Materials and Methods), as found in PFC neurons (Haj-Dahmane and Andrade, 1997).

\section{$\mathrm{CB}$ is robust using the event/delay protocol}

To assess the robustness of $\mathrm{CB}$ mnemonic properties, we parametrically explored the model in response to the event/delay protocol (Fig. 2a), as a function of $I_{\text {Inj-delay }}$ and $g_{\text {CAN }}$, which is important because it reflects the regulation history of spikemediated excitability and dictates the possible existence of $\mathrm{CB}$ (Fig. 1). We found that $\mathrm{CB}$ existed in a large range of $g_{C A N}$ values (Fig. 2a, $\mathrm{CB}$ domain). Moreover, the ranges of $\mathrm{CB}$ and $\mathrm{AB}$ domains were much wider than $M$ domains, indicating the prevalence of mnemonic properties with spike-mediated excitability in the model. We also found that cellular memory expressed differentially, depending on delay stimulation conditions. In the $g_{\text {CAN }}$ range of $\mathrm{CB}$, there was no firing during the delay at the lowest $I_{\text {Inj-delay }}$ values (i.e., discharge was memoryless; Fig. $2 a$, yellow domain and trace). In contrast, delay firing was slowly decaying in a significant $I_{\text {Inj-delay }}$ range below $\theta_{\mathrm{OFF}}$, underlying a transient memory (Fig. $2 a$, orange), whereas above $\theta_{\text {OFF }}$ a stable conditional memory was observed (compare Figs. $2 a$, lavender, $1 c$ ). In addition, we observed a stable absolute memory (i.e., sustained activity without self-termination) in the range of $\mathrm{AB}$ (compare Figs. $2 a$, purple, $1 b$ ). Under $\mathrm{CB}$, memory typically lasted hundreds of milliseconds when transient (Fig. $2 b$ ) and firing frequency was generally moderate $(<50 \mathrm{~Hz})$, in contrast to $\mathrm{AB}$ (Fig. $2 c$ ). These results indicated that spike-mediated CB is robust, multiform, with long durations and low frequencies, which is consistent with persistent activity in the PFC during WM tasks (Compte, 2006). Moreover, parametrically, CB lies between $M$ and $A B$, which have both largely been observed (Haj-Dahmane and Andrade, 1997; Dembrow et al., 2010; Gee et al., 2012). This suggests that $\mathrm{CB}$, although rarely observed in the PFC (Thuault et al., 2013), may have been previously overlooked, because the event/delay protocol, which is mandatory to reveal it, is almost never used in intracellular recordings.

\section{CB generically emerges from spike-mediated excitability}

We wondered whether $\mathrm{CB}$ mnemonic properties were generic in essence, or specific to the model considered. Spike-mediated biophysical determinants- $\mathrm{CaL}$ and CAN current gating variables and the intracellular calcium $\left[\mathrm{Ca}^{2+}\right]$-share a common dynamical trait. Their spike-triggered activation operates faster, compared with 

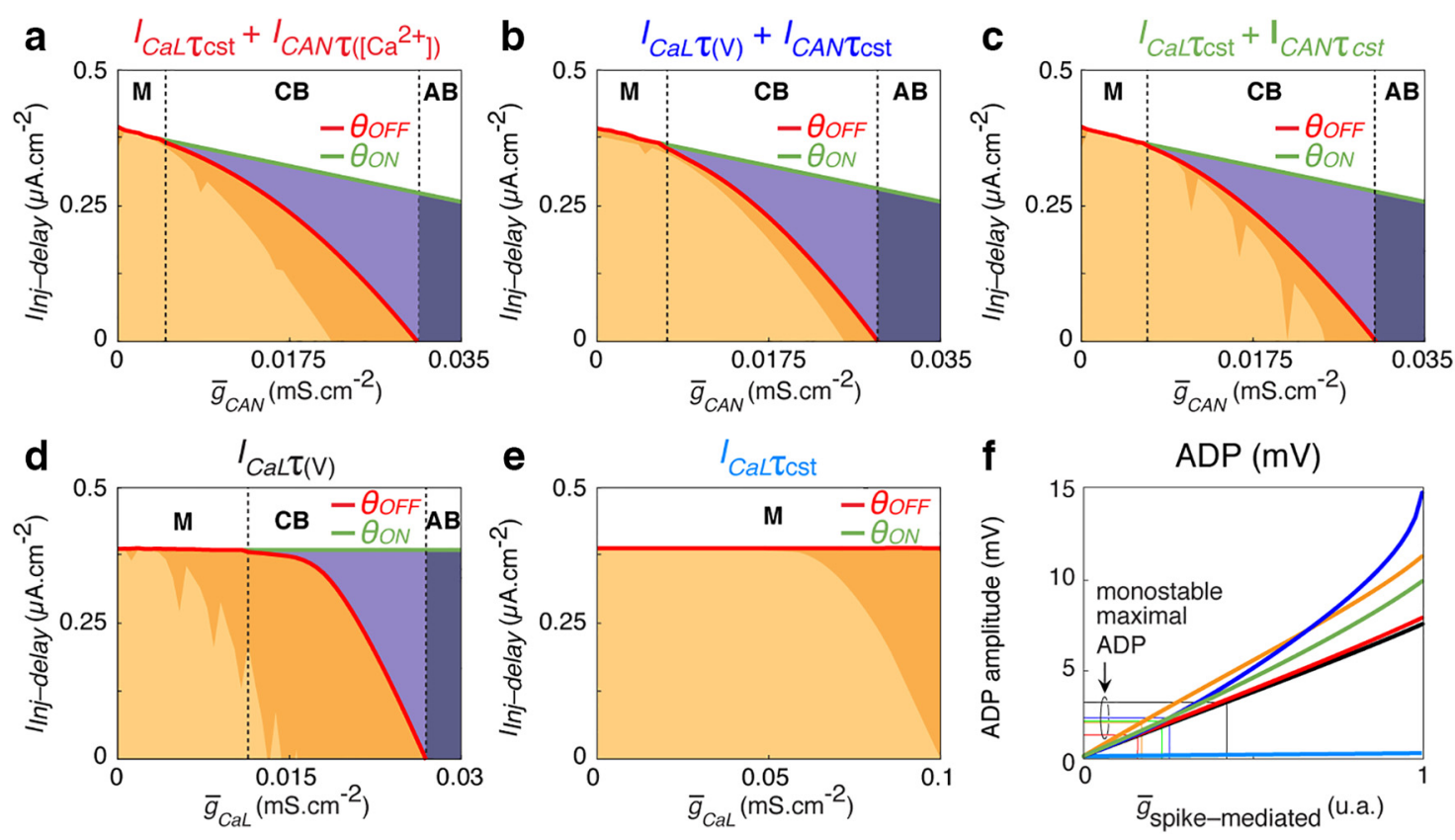

Figure 3. Conditional bistability is a generic mnemonic property of neurons endowed with depolarizing spike-mediated mechanisms. $\boldsymbol{a}-\boldsymbol{e}$, Thresholds for initiating ( $\theta_{\mathrm{ON}}$, green lines) and terminating spiking ( $\theta_{\text {OFF, }}$ red lines) for models with fixed (i.e., voltage- or calcium-independent) time constants suppressing the dynamic asymmetry of the CaL current (a), the CAN current ( $\boldsymbol{b}$ ), or both $(\boldsymbol{c})$, or for models endowed with the sole CaL current with a voltage-dependent (d) or a fixed ( $\boldsymbol{e}$ ) time constant as a function of the suprathreshold maximal conductance and background delay current. $\boldsymbol{f}, A D P$ amplitudes of the five alternative models presented in $\boldsymbol{a}-\boldsymbol{e}$. Line colors match the respective panel titles, and the orange line applies to the standard model. Note that for ADP amplitudes $(\boldsymbol{f})$ suprathreshold maximal conductances were normalized by the boundary value defining the transition between conditional and absolute bistability for each model. $M$, $C B$, and $A B$ indicate the monostability, conditional bistability, and absolute bistability domains, respectively. Sawteeth at the border between memoryless and transient regions correspond to the discharge of one spike occurring at $>25 \mathrm{~ms}$ after the onset of the delay period, while the main part of the transient memory region corresponds to the discharge of several spikes during the delay period (see definition of transient memory behavior in Materials and Methods).

their relaxation timescale during the ISI. This asymmetry produces interspike traces that form a memory after each spike, favoring the firing of the following spike and, in turn, self-sustained spiking. We tested whether the dynamic asymmetry of these determinants was essential to cellular memory. We found that considering fixed (i.e., voltage- or calcium-independent) time constants to suppress the dynamic asymmetry of the CaL, the CAN, or both currents had no effect on cellular memory (compare Figs. $3 a-c, 2 a$ ), indicating that $\left[\mathrm{Ca}^{2+}\right]$ dynamic asymmetry was sufficient to support cellular memory. We also found that, in the absence of both calcium dynamics and the CAN current, CaL asymmetry alone was both sufficient (Fig. 3d) and necessary (Fig. 3e). Therefore, while cellular memory required the asymmetry between activation/relaxation time constants of a least one determinant, it was independent of its exact nature. This demonstrated that dynamic asymmetry was generic in underlying the positive feedback of spike-mediated CB. Remarkably, we found that CB coexisted with marked ADP amplitudes ( $\sim 2.5$ to $15 \mathrm{mV}$ ) after spiking (Fig. $3 f$, above horizontal lines), contrasting with the smaller ADP of monostable neurons $(<2.5 \mathrm{mV})$.

\section{$\mathrm{CB}$ mnemonic properties under in vivo conditions}

In vivo, PFC neurons continuously receive asynchronous synaptic inputs inducing strong membrane-potential fluctuations. These fluctuations may disrupt conditional memory, which relies on a minimal subthreshold depolarization. Thus, we assessed cellular memory with stochastic synaptic excitatory (AMPA) and inhibitory $\left(\mathrm{GABA}_{\mathrm{A}}\right)$ inputs driving fluctuations as found in vivo in the PFC (i.e., several millivolts; Fellous et al., 2003). Here, we tested the response of the neuron to the protocols considered in vitro and to a delay protocol (i.e., devoid of event). The latter was used as a control, since stochasticity may induce spiking during the subthreshold delay input. We found that at $g_{\text {CAN }}$ levels providing $\mathrm{CB}$ in vitro, the neuron responded in vivo to the event/ delay protocol with a persistent activity (Fig. $4 a$, right) that was absent after the event protocol (left) and initially weaker during the delay protocol (middle). As a general rule, activity included episodes during which spikes clustered in bursts and spikemediated currents were significantly activated (Fig. $4 a$, lavender). During bursting episodes, the positive feedback characterizing $\mathrm{CB}$ ensured self-sustained spiking, which was irregular and terminated because of synaptic fluctuations. Bursting episodes alternated with nonbursting episodes essentially characterized by single spiking at lower frequency and smaller spike-mediated current activation (Fig. $4 a$, yellow; i.e., during which the positive feedback was disengaged). A raster plot across trials (Fig. 4b) illustrates stronger activity, a larger bursting propensity, and important variability in the temporal structure of the discharge during the event/delay protocol.

While firing slowly increased during the delay protocol (Fig. $4 c$, fuschia) and rapidly decayed after the event protocol (Fig. $4 c$, light blue), it persisted longer during the delay in the event/delay protocol (Fig. $4 c$, lavender; $\tau_{\text {memory }} \sim 900 \mathrm{~ms}$ ), with a frequency exceeding the sum of firing frequencies triggered by event or delay inputs alone (Fig. $4 c$, salmon). Thus, persistent activity is an emergent property arising from nonlinear interactions between spike-mediated currents and the delay background input. Persistent activity with $\tau_{\text {memory }}$ in the range of hundreds of milliseconds to seconds (i.e., consistent with WM) was robustly evoked for a large domain of event input parameters (Fig. $4 d$ ) and a thinner domain of the delay input parameters (Fig. 4e). Large $\tau_{\text {memory }}$ values were observed when the event was stronger than the delay 
a $V(\mathrm{mV})$
$x_{C a L}$ (a.u.)
$\left[\mathrm{Ca}^{2+}\right](\mu \mathrm{M})$
$x_{C A N}($ a.u. $)$
$x_{A H P}($ a.u. $)$
$g_{i}(t)$
$g_{\theta}(t)$ non-bursting

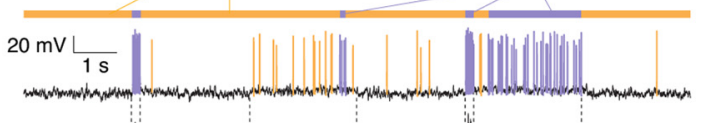

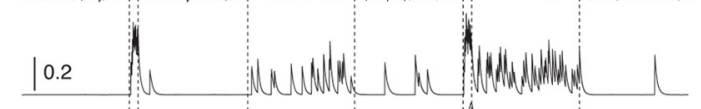
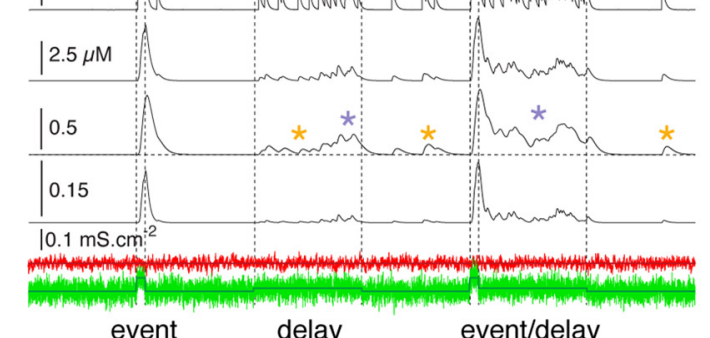

event

delay

b

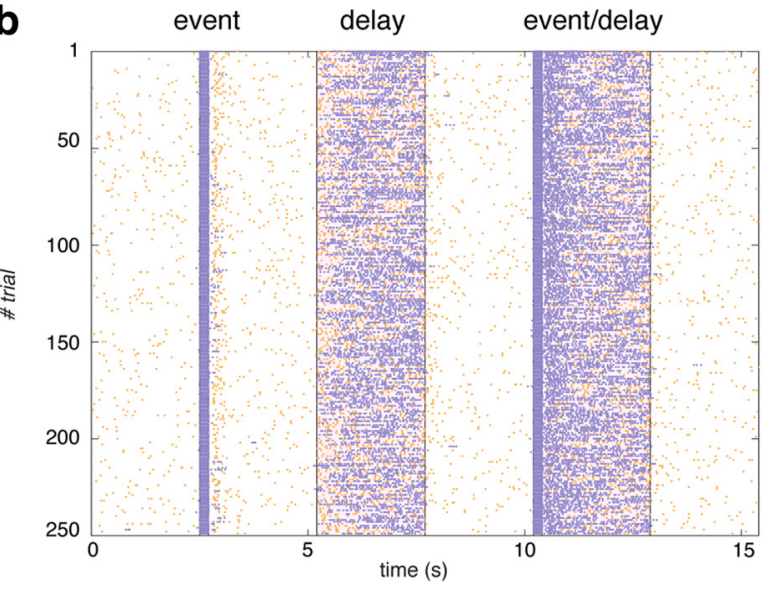

C frequency post-stimulus histogram

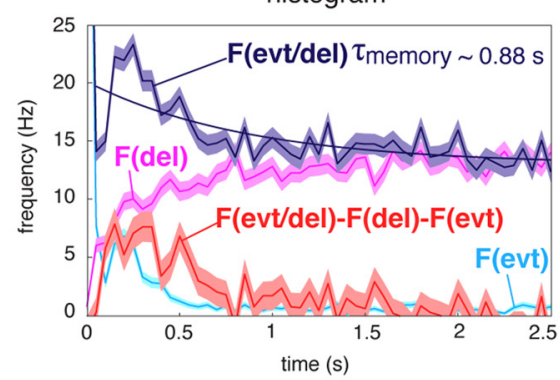

d

d $\quad \tau$ memory $=\mathrm{f}$ (event) (event/delay protocol)

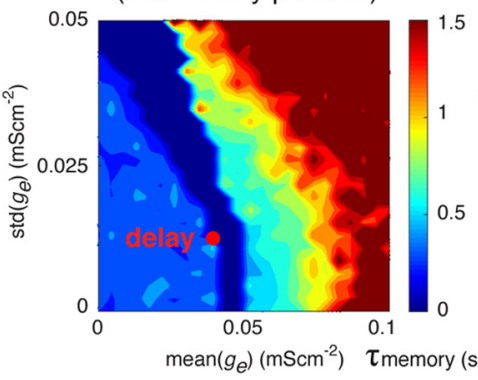

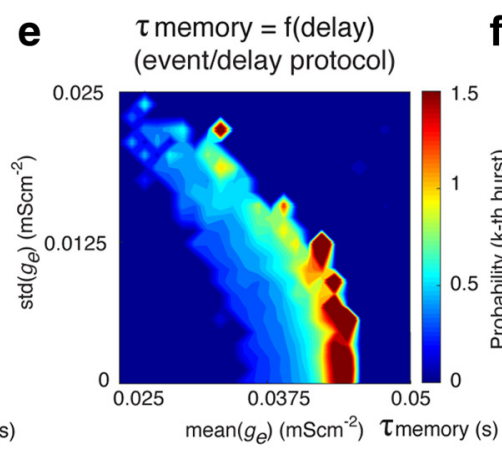

f onset times

of bursting episodes
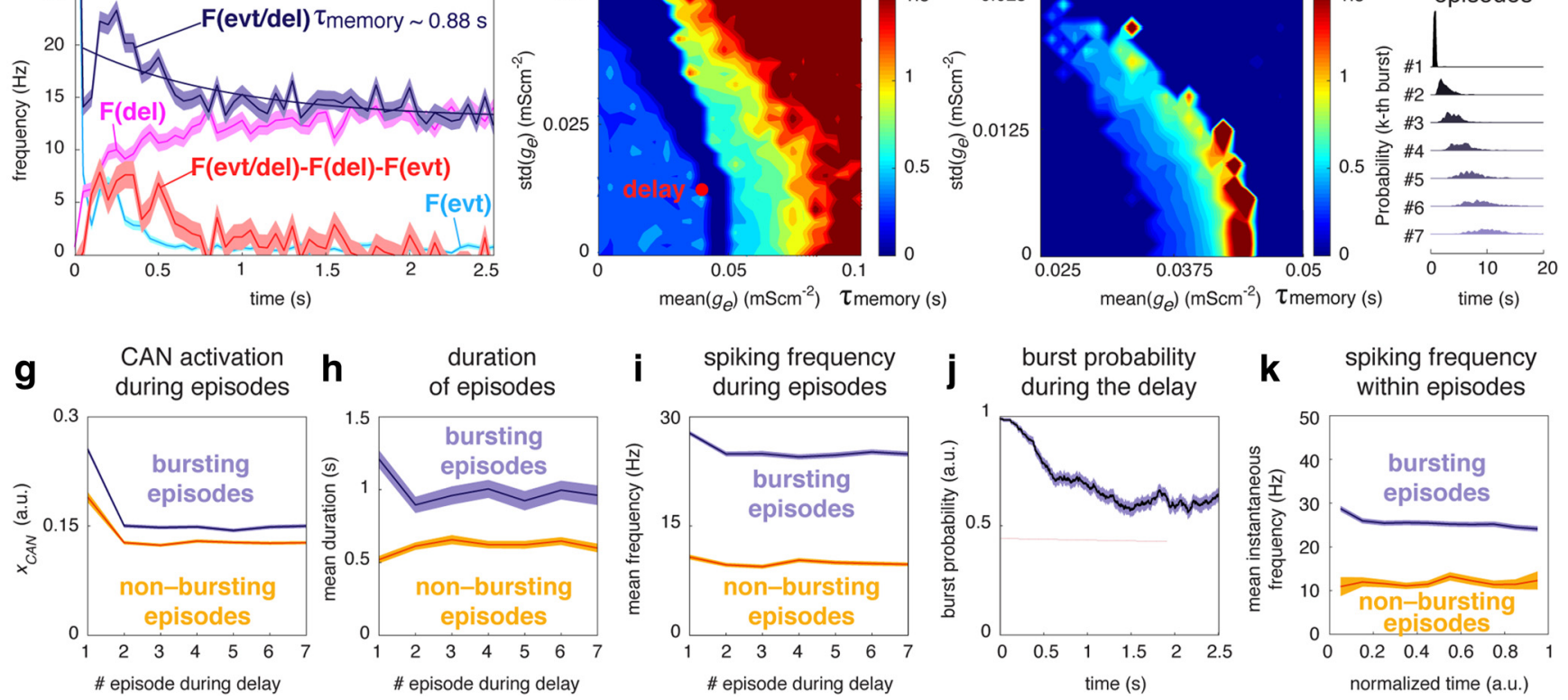

Figure 4. Conditional bistability confers robust event memory under strongly fluctuating synaptic inputs in vivo. $\boldsymbol{a}$, Membrane potential, CaL and CAN activation, calcium dynamics, and AHP activation traces of the standard neuron model in response to the event, delay, and event/delay protocols for a realization of the excitatory (green) and inhibitory (red) synaptic fluctuating conductances. Spikes belonging to bursting and nonbursting episodes are indicated in lavender and yellow, respectively. Small activation buildups of spike-mediated currents during nonbursting episodes and larger buildups during bursting episodes are signaled by yellow and lavender stars, respectively. See Materials and Methods for criteria that define bursting episodes. $\boldsymbol{b}$, Spike raster plot for 250 trials of the protocol depicted in $\boldsymbol{a}$, with different realizations of synaptic fluctuations. Color code as in $\boldsymbol{a}$. c, Frequency poststimulus time histogram (PSTH) of the discharge (250 trials) after the onset of event (light blue), delay (fuschia), and event/delay (lavender) protocols; difference between the PSTH during the event/delay protocol, and the sum of PSTHs during the event and delay protocols (salmon). The mean \pm SEM frequency values are displayed. The memory time constant is defined as the time constant of firing frequency relaxation to its steady-state value in the event/delay protocol. $\boldsymbol{d}$, Memory time constant map of persistent activity, as a function of the mean and SD of the excitatory fluctuating conductance of the event input during the event/delay protocol. The red dot indicates conductance parameters of the delay background input. When the event mean conductance is smaller than that of the delay input (left part of the map), the activity builds up to the steady state during the delay from the lower event trigger initial frequency (e.g., pink curve in c, for a null event mean conductance) and the time constant is smaller.e, Memory time constant map of persistent activity, as a function of the mean and SD of the excitatory fluctuating conductance of the delay input during the event/delay protocol. $\boldsymbol{d}-\boldsymbol{e}$, Means across 100 trials; other synaptic parameters as in the standard model (see Materials and Methods). $f$, Probability distribution of onset times of bursting episode as a function of their order of occurrence during the delay period (20 s) of the event/delay protocol, across 250 trials. $\boldsymbol{g}-\boldsymbol{i}$, CAN conductance activation $(\boldsymbol{g})$, mean duration $(\boldsymbol{h})$, and mean spiking frequency (i) during bursting (lavender) and nonbursting (yellow) episodes, as a function of their order of occurrence during a delay period ( $20 \mathrm{~s}$ ) in the event/delay protocol. Mean \pm SEM values across 250 trials. $j$, Probability of being in a burst episode during the delay period ( $2.5 \mathrm{~s}$ ) in the event/delay protocol. $\boldsymbol{k}$, Mean instantaneous spiking frequency as a function of the normalized time within bursting and nonbursting episodes (normalized time equals 0 at the beginning of episodes, 1 at their end). Mean \pm SEM values across 100 trials.

(Fig. $4 d$, right part of the map), with persistent activity decaying during the delay (Fig. 4c, lavender).

Mechanistically, the excitation provoked by the event favored the rapid engagement of the positive feedback during the delay, as reflected by the strong synchronization of the onset of the first bursting episode across trials (Fig. 4f). This first episode displayed a larger recruitment of spike-mediated currents (Fig. $4 g$ ) and an increased duration (Fig. 4h) and frequency (Fig. 4i), compared with the following bursting episodes. As a result, the probability of being in a bursting episode (i.e., at a higher firing frequency) remained high at the beginning of the delay and progressively decreased toward its steady state (Fig. 4j), accounting for the decreasing pattern of firing frequency (Fig. $4 c$, lavender). Note that the instantaneous firing frequency remained globally 

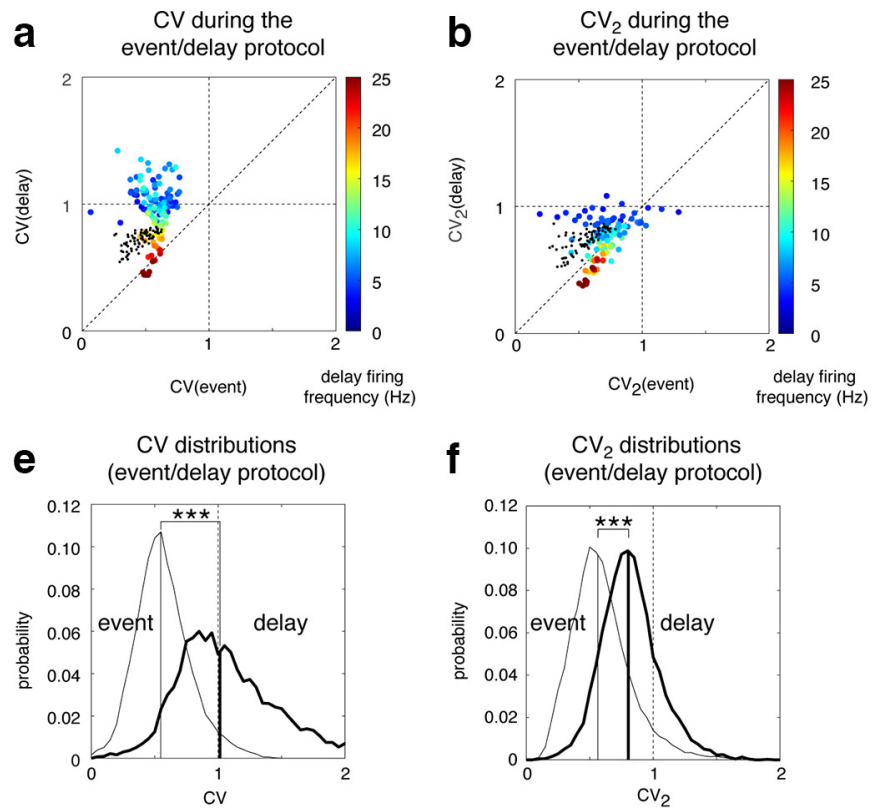
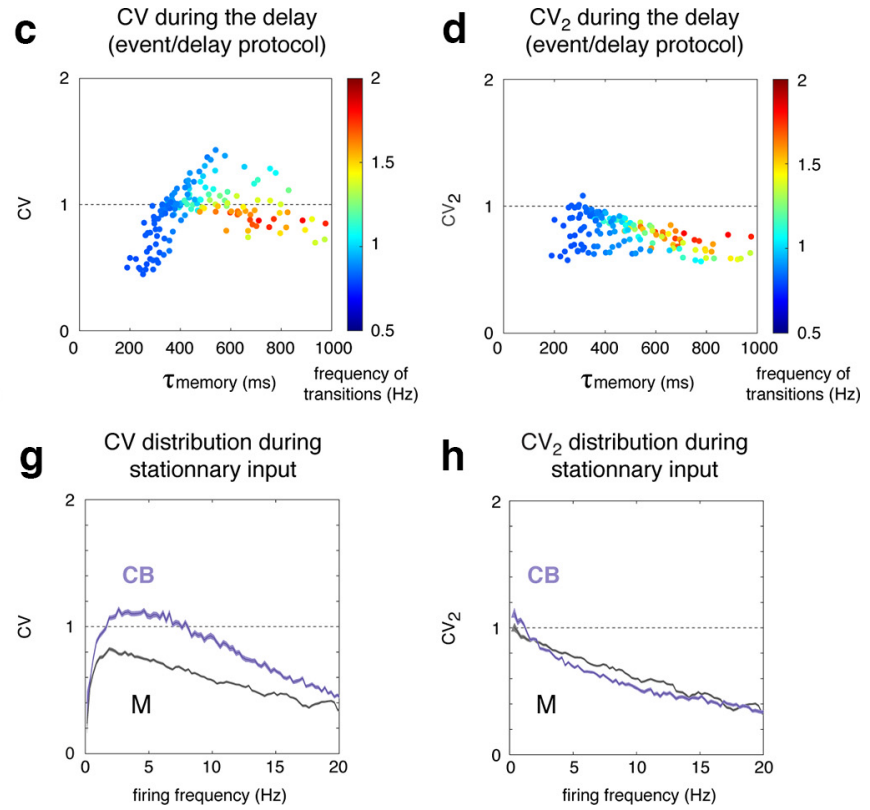

Figure 5. Conditional bistability promotes irregular discharge under in vivo conditions. $\boldsymbol{a}, \boldsymbol{b}, \mathrm{CV}(\boldsymbol{a})$ and $\mathrm{CV}_{2}(\boldsymbol{b})$ measures of the ISI distribution of the discharge in monostable (black dots; $g_{\text {CAN }}=0 \mathrm{~ms} \cdot \mathrm{cm}^{-2}$ ) and conditionally bistable (colored dots; $g_{\text {CAN }}=0.025 \mathrm{mS} \cdot \mathrm{cm}^{-2}$ ) neurons, in response to the input of an event protocol ( $x$-axis) and to the $2.5 \mathrm{~s}$ delay input of an event/delay protocol ( $y$-axis). The color code for conditionally bistable neurons indicates the firing frequency during the delay. Both inputs have the same excitatory input parameter taken in the ranges $0-0.05$ $\mathrm{mS} \cdot \mathrm{cm}^{-2}$ for the mean and $0-0.025 \mathrm{mS} \cdot \mathrm{cm}^{-2}$ for the SD, to limit the effect of firing frequency, which affects $\mathrm{CV} / \mathrm{CV}_{2}$ measures in a nontrivial fashion (Compte et al., 2003). In both protocols, the event input lasts $0.5 \mathrm{~s}$, as in the study by Compte et al. (2003). In the event/delay protocol, the event input has a $0.065 \mathrm{mS} \cdot \mathrm{cm}^{-2}$ mean and a $0.0125 \mathrm{mS} \cdot \mathrm{cm}^{-2} \mathrm{SD}$. $\boldsymbol{c}, \boldsymbol{d}$, In the conditionally bistable neuron, the highest discharge irregularity during the delay, measured by the $\mathrm{CV}\left(\boldsymbol{c} ; y\right.$-axis) and the $\mathrm{CV}_{2}(\boldsymbol{d} ; y$-axis) is observed at intermediate memory time constants ( $300-600 \mathrm{~ms}$; $x$-axes) and frequencies of transitions between bursting and nonbursting episodes (color code). $\boldsymbol{e}, \boldsymbol{f}$, At moderate memory time constants ( $400-600 \mathrm{~ms})$, the means of $\mathrm{CV}_{(\boldsymbol{e})}$ ) and $\left(V_{2}(\boldsymbol{f})\right.$ probability density functions during the delay are significantly higher in the (B neuron during the delay (thick black curve), compared with those during the event (thin black curve); $p<1 \mathrm{e}^{-9}\left({ }^{* * *}\right)$ on two-tailed Wilcoxon rank-sum tests for both the CV and CV 2 distributions (distributions were not normal, according to Kolmogorov-Smirnov goodness-of-fit hypothesis tests; for the CV distribution: $n_{\text {event }}=29,092, n_{\text {delay }}=5448$, median $\left(\mathrm{CV}_{\text {event }}\right)=0.5478$, and median $\left(\mathrm{CV}_{\text {delay }}\right)=1.0142 ;$ for the $\mathrm{CV}_{2}$ distribution: $n_{\text {event }}=26,755, n_{\text {delay }}=5345$, median $\left(\mathrm{CV}_{2, \text { event }}\right)=0.5634$, and median $\left.\left(\mathrm{CV}_{2, \text { delay }}\right)=0.8043\right) \cdot \boldsymbol{g}, \boldsymbol{h}$, Irregularity of the discharge plotted as a function of firing frequency for the conditionally bistable (lavender) and monostable neurons (black) in response to stationary synaptic inputs, as measured by the CV $(\boldsymbol{g})$ and the $\mathrm{CV}_{2}(\boldsymbol{h})$.

constant within episodes (i.e., the discharge was quasi-stationary; Fig. 4k).

\section{CB promotes irregular discharge under in vivo conditions}

In WM tasks, spiking irregularity is larger during the delay than during stimulus presentation (i.e., event; Compte et al., 2003), with a higher coefficient of variation (CV) of ISIs over 1 and a $\mathrm{CV}_{2}$ (a version of CV based on successive ISIs) of $\sim 1$, which has been difficult to reproduce robustly in theoretical models (Barbieri and Brunel, 2008). In our model, irregularity was generally higher during the delay, compared with the event (Fig. $5 a, b$, dots above first bisector), independently of whether neurons were $\mathrm{M}$ (Fig. $5 a, b$, no $I_{\mathrm{CAN}}$, black dots) or CB (Fig. $5 a, b$, colored dots). Indeed, at a given similar firing mean frequency, the longer delay $(2.5 \mathrm{~s})$ allowed longer ISIs that could not occur during the shorter event $(0.5 \mathrm{~s})$. Thus, during the event, the sampling of the ISI distribution was truncated at low frequencies, and the apparent ISI variance was therefore decreased, compared with the delay. This effect was moderate for $\mathrm{M}$ neurons (Fig. $5 a, b$, black dots), but it dramatically increased for CB neurons firing at low frequency ( $<15 \mathrm{~Hz}$; Fig. $5 a, b$, colored dots), since, in the latter neurons, alternations of bursting episodes (with smaller ISIs) and nonbursting episodes (with larger ISIs) strongly increased the variance of the ISI distribution during the delay. At such lowfrequency firing, the $C V$ was largely $>1$ and the $\mathrm{CV}_{2}$ was $\sim 1$, as found during WM delays (Compte et al., 2003). Moreover, CV/ $\mathrm{CV}_{2}$ culminated for inputs leading to intermediate memory time constants in the range of $\sim 400-600 \mathrm{~ms}$ (Fig. $5 c, d$ ) and transition frequencies between episodes at $\sim 1 \mathrm{~Hz}$ (color code). In these conditions, both the $\mathrm{CV}$ and $\mathrm{CV}_{2}$ were significantly larger during the delay (Fig. $5 e, f$, thick black trace), compared with the event (Fig. 5e,f, thin black trace). Remarkably, consistent with data (Compte et al., 2003), the CV distribution during the delay was broadened, compared with that during the event, which did not occur for the $\mathrm{CV}_{2}$.

To fully confirm the genuine effect of depolarizing spikemediated currents on spiking irregularity, we compared $\mathrm{CV} / \mathrm{CV}_{2}$ with and without $\mathrm{CB}$ (1) upon stationary stimuli to avoid the interference of frequency time variations due to the protocol and (2) at identical mean firing frequencies to avoid the nontrivial effects of frequency on these measures (Compte et al., 2003). In these conditions, where computing these observables admits its plain significance, we found that, compared with $\mathrm{M}$ neurons (Fig. $5 g$, black), the $\mathrm{CV}$ was systematically superior in $\mathrm{CB}$ neurons (Fig. $5 g$, lavender) $<20 \mathrm{~Hz}$ and was $>1$ below $10 \mathrm{~Hz}$. In CB neurons, the $\mathrm{CV}_{2}$ was also superior below $2 \mathrm{~Hz}$, situated at $\sim 1$, whereas it was essentially similar to $\mathrm{M}$ neurons $>2 \mathrm{~Hz}$ (Fig. $5 h$ ). Thus, although the mean local irregularity measured by the $\mathrm{CV}_{2}$ was the same on average (because local increases of frequency regularity within bursting episodes compensated for the local frequency irregularities at transitions between bursting and nonbursting episodes), we found that the global irregularity of the discharge (measured by the CV; i.e., the normalized ISI SD) was increased in $\mathrm{CB}$ neurons due to the presence of spike-mediated currents.

\section{Discussion}

Here, we show that spike-mediated CaL and CAN currents of L5 pyramidal PFC neurons (Haj-Dahmane and Andrade, 1997; Gee 
et al., 2012; Thuault et al., 2013) support CB. Moreover, our study suggests that $\mathrm{CB}$ is prevalent for several reasons. First, $\mathrm{CB}$ relies on suprathreshold mechanisms that are ubiquitous in pyramidal PFC neurons and operate robustly, independent of biophysical details, which are generic. Second, CB parametrically situates between $\mathrm{M}$ and $\mathrm{AB}$ regimes, both extensively observed in the PFC and other areas (Krnjević et al., 1971; Schwindt et al., 1988; Yang et al., 1996; Haj-Dahmane and Andrade, 1997; Dembrow et al., 2010; Zhang and Séguéla, 2010; Gee et al., 2012). AB is often observed under strong neuromodulatory manipulation that upregulates depolarizing spike-triggered conductances, yielding unrealistic stereotyped discharges inconsistent with WM firing patterns (Compte et al., 2003; Shafi et al., 2007). This suggests that neuromodulation regulates conductances below the range for $\mathrm{AB}$ in behaving animals. Below $\mathrm{AB}$, the probability of being in $\mathrm{CB}$ is largely predominant (a much wider range than M; Fig. 2a). Moreover, minimal neuromodulation is crucial for optimal PFC computations (Wang et al., 2007), whereas M lies at the lowest neuromodulation (conductance) levels. Thus, CB is likely encountered in PFC pyramidal neurons under physiological neuromodulatory levels. Third, we show that ADP represents a generic marker distinguishing $\mathrm{CB}$ from $\mathrm{M}$ neurons. ADPs are ubiquitous across L5 PFC pyramidal types (Yang et al., 1996; Haj-Dahmane and Andrade, 1997) and share specific common features with CB neurons ( $>5 \mathrm{mV}$; durations up to $\sim 100 \mathrm{~ms}$, occurrence even at low frequencies, CAN/calcium dependence; Yang et al., 1996; Haj-Dahmane and Andrade, 1997; Boudewijns et al., 2013), suggesting that PFC neurons displaying ADP are conditionally bistable. Hence, CB was observed without artificial pharmacological activation in L5 PFC pyramidal neurons with prominent ADPs (Thuault et al., 2013). Altogether, these lines of evidence indicates that $\mathrm{CB}$ likely constitutes a prevalent property in PFC L5 pyramidal neurons in physiological conditions during WM tasks.

So, why has $\mathrm{CB}$ remained scarce in the PFC? CB requires a triggering suprathreshold input followed by a subthreshold input (or applied upon a depolarized subthreshold holding potential). Therefore, CB neurons are undetectable using the classic protocol ubiquitously used, which consists of a single suprathreshold input applied from the resting potential. Consequently, neurons can be categorized as M (Fig. 1c1), while actually displaying genuine CB (Fig. 1c2). Such misclassification should be frequent given the much larger $\mathrm{CB}$ domain (compared with $\mathrm{M}$ ), and systematically applying event/delay protocols should unravel CB in a significant fraction of neurons. Remarkably, event/delay protocols are meaningful physiologically, mimicking the temporal profile of inputs during WM: a strong behaviorally relevant (e.g., perceptive) signal followed by a lower background input during the delay (e.g., reverberating persistent activity or WM-related feedforward inputs).

Information maintenance in CB neurons relies on the asymmetry between fast buildup/activation and slower relaxation/ deactivation dynamics of spike-activated mechanisms. This asymmetry maintains spike-to-spike excitability through the positive feedback between depolarization and suprathreshold activation. Noticeably, the slow CAN deactivation time constant of $\sim 100$ ms (Haj-Dahmane and Andrade, 1997) allows the maintenance of self-sustained activity down to $\sim 10 \mathrm{~Hz}$. Slower CAN in the PFC (Sidiropoulou et al., 2009) may support lower frequencies, at some expense (see below). Under asynchronous inputs, $\mathrm{CB}$ generates bursting/nonbursting episodes. Bursting episodes can be triggered by - and form a memory of-incoming events, when a background input follows the event. Statistically, this per- sistent activity fades at the second timescale across trials, consistent with WM, reflecting the stochastic disruption of bursting episodes due to synaptic fluctuations. By contrast, firing frequency is steady within bursting episodes, so that information maintenance is constant within individual trials for the duration of the first bursting episode.

Overall, the spike-mediated mechanism we unravel is robust to the exact nature and parameter values of the model and displays a much higher resistance to transient episodes of inhibition, compared with subthreshold-based bistabilities (Washburn et al., 2000; Loewenstein et al., 2005; Carrillo-Reid et al., 2009). Sensitivity to inhibitory interference was used to discard the possible role of intrinsic bistability in maintaining persistent activity (Sanchez-Vives and McCormick, 2000; McCormick et al., 2003). Our results indicate that this reasoning does not apply to spikemediated mechanisms, because they preserve resistance to inhibition, as does synaptic reverberation.

Previously described bistabilities are rigid, requiring strong/ long stimuli to be turned on/off and producing long, high-frequency discharges primarily independent of the background input (HajDahmane and Andrade, 1997; Egorov et al., 2002; Tahvildari et al., 2007; Zhang and Séguéla, 2010; Gee et al., 2012) By contrast, $\mathrm{CB}$ exhibits a rich repertoire of computational operations. It expresses as a memoryless discharge or subserve transient or stable conditional memory, depending on input parameters. Moreover, mnemonic activities can be initiated by short events because of the moderate CAN activation time constant (Haj-Dahmane and Andrade, 1997). Furthermore, the duration and frequency of mnemonic discharges are controlled by the delay input at low frequencies. Finally, under in vivo-like inputs, this diversity expresses as bursting/nonbursting episodes with variable frequencies and durations, resulting in a large variability of the discharge structure across trials, as found during WM (Shafi et al., 2007).

In response to asynchronous inputs, $\mathrm{CB}$ increases discharge irregularity, because smaller ISIs during bursting episodes and larger ISIs during nonbursting episodes increase the ISI distribution variance. The $\mathrm{CV} / \mathrm{CV}_{2}$ are highest at low firing frequencies i.e., under excitation/inhibition balance, two factors increasing irregularity; Compte et al., 2003. In such conditions, the CV is $>1$ and $\mathrm{CV}_{2} \sim 1$ in $\mathrm{CB}$ neurons during the delay, being higher than during the event, as in WM (Compte et al., 2003), properties previous models are unable to account for robustly (Barbieri and Brunel, 2008). This effect happens in CB neurons, because bursting/nonbursting episodes can alternate during delays of several seconds, but not during shorter events $(0.2 \mathrm{~s})$.

Besides, while $\mathrm{CB}$ clearly increases the $\mathrm{CV}$, its effect on the $\mathrm{CV}_{2}$ is mild. This results because whereas frequency changes at the transitions of episodes increase $\mathrm{CV}_{2}$, the more regular discharge within bursting episodes decreases it. Synaptic inputs are not stationary in vivo (Shafi et al., 2007; Ostojic, 2014), which could explain the slightly higher $\mathrm{CV} / \mathrm{CV}_{2}$ observed experimentally (Compte et al., 2003), compared with the situation reported here. This could also explain the larger difference in $\mathrm{CV}_{2}$ values between the delay and the event (Compte et al., 2003), as changes in the synaptic input rates have more time during the delay to exert their effect on successive ISIs and thus on the $\mathrm{CV}_{2}$.

Interestingly, the overall increase in irregularity in $\mathrm{CB}$ neurons required an AHP current, which balanced the CAN current in the model (CAN alone decreased irregularity; data not shown). Finally, our conclusion that busting/nonbursting alternations underlie irregularity is additionally supported by the finding that very slow CAN currents-driving very long 
bursts without alternations-decrease the CV (Sidiropoulou et al., 2009).

What roles may $\mathrm{CB}$ play at the network scale during maintenance? Here, $\mathrm{CB}$ requires a subthreshold constant background input from the network to memorize a transient event. However, inputs are not stationary in PFC networks and cellular CB should affect, in turn, network dynamics. Therefore, interactions between local cellular CB and global network recurrence may provide a rich repertoire of dynamics.

Hence, following an event, bursting in CB neurons may be sustained by the prolonged synaptic feedback due to bursting in other CB neurons. Such synergistic CB bursting recruitment may determine the extent to which activity is amplified and prolonged, possibly resulting in decaying, stable, or ramping temporal firing patterns of WM (Shafi et al., 2007). Synergy between CB neurons may also provide a realistic biophysical basis for WM of parametric information, which requires bistable elements to emerge robustly (Koulakov et al., 2002; Goldman et al., 2003). Such collective dynamics are plausible because $\mathrm{CB}$ is gradual in essence, by contrast to $\mathrm{AB}$. WM-related drives during the delay and the regulation state of synaptic strengths and spike-mediated excitability should be fundamental in setting the gradual synergetic recruitment of $\mathrm{CB}$ neurons.

Within a recurrent network, $\mathrm{CB}$ neurons can discharge during the delay even when they have not received the event input, because of the subthreshold recurrent input provided by other neurons of the network actively maintaining the memory of that event (Fig. $4 c$, fuschia curve). This could be problematic if presynaptic and postsynaptic neurons belong to different populations encoding distinct memories (i.e., Hebbian assemblies), as memory would "bleed over" across populations (i.e., memory interference). This problem may arise even with monostable postsynaptic neurons, although $\mathrm{CB}$ neurons would discharge at higher rates for a similar recurrent delay input, enhancing interference. However, different mechanisms have been imagined that may circumscribe interference between memory representations [e.g., mutual (Miller and Wang, 2006) or global (Brunel and Wang, 2001) inhibition between assemblies]. Besides, enhanced "bleeding" due to CB could also improve pattern completion within Hebbian assemblies, because the easier recruitment of $\mathrm{CB}$ neurons not activated by the event (because of incomplete input pattern presentation) would facilitate complete memory retrieval through associative synaptic reverberation.

Besides, during WM delays, PFC networks encounter transitions between stable collective states of quasi-stationary firing at the second timescale, reflecting mental states during the exploration of computational solutions, as cognitive processes wander from stimulus encoding to decision-making and action (Seidemann et al., 1996; Cossart et al., 2003). Bursting/nonbursting episodes in CB neurons share similar quasi-stationary firing and generate maximal irregularity at this timescale. We suggest that $\mathrm{CB}$ may promote the emergence of stable collective states and the complexity of PFC neuronal operations, providing a basis for exploring computational solutions during WM. Intrinsic plasticity and neuromodulation would represent strategic processes to regulate spike-mediated mechanisms for the emergence of adapted WMrelated cognitive processes.

While $\mathrm{CB}$ relies on a weak spike-mediated positive feedback, it is precisely this "weakness" that underpins the computational richness and flexibility it brings, compared with what was previously thought. We suggest that the traditional view should be overcome in favor of a reconciling perspective whereby synaptic reverberation and conditional bistability concur with the emer- gence of the highly flexible persistent activity required for elaborating adaptive WM-related cognitive processes and intelligent behavior.

\section{References}

Barbieri F, Brunel N (2008) Can attractor network models account for the statistics of firing during persistent activity in prefrontal cortex? Front Neurosci 2:114-122. CrossRef Medline

Booth V, Rinzel J (1995) A minimal, compartmental model for a dendritic origin of bistability of motoneuron firing patterns. J Comput Neurosci 2:299-312. CrossRef Medline

Boudewijns ZS, Groen MR, Lodder B, McMaster MT, Kalogreades L, de Haan R, Narayanan RT, Meredith RM, Mansvelder HD, de Kock CP (2013) Layer-specific high-frequency action potential spiking in the prefrontal cortex of awake rats. Front Cell Neurosci 7:99. CrossRef Medline

Bourque CW (1986) Calcium-dependent spike after-current induces burst firing in magnocellular neurosecretory cells. Neurosci Lett 70:204-209. CrossRef Medline

Brunel N, Wang XJ (2001) Effects of neuromodulation in a cortical network model of object working memory dominated by recurrent inhibition. J Comput Neurosci 11:63-85. CrossRef Medline

Camperi M, Wang XJ (1998) A model of visuospatial working memory in prefrontal cortex: recurrent network and cellular bistability. J Comput Neurosci 5:383-405. CrossRef Medline

Carrillo-Reid L, Tecuapetla F, Vautrelle N, Hernández A, Vergara R, Galarraga E, Bargas J (2009) Muscarinic enhancement of persistent sodium current synchronizes striatal medium spiny neurons. J Neurophysiol 102: 682-690. CrossRef Medline

Compte A, Constantinidis C, Tegner J, Raghavachari S, Chafee MV, Goldman-Rakic PS, Wang XJ (2003) Temporally irregular mnemonic persistent activity in prefrontal neurons of monkeys during a delayed response task. J Neurophysiol 90:3441-3454. CrossRef Medline

Compte A (2006) Computational and in vitro studies of persistent activity: edging towards cellular and synaptic mechanisms of working memory. Neuroscience 139:135-151. CrossRef Medline

Cossart R, Aronov D, Yuste R (2003) Attractor dynamics of network UP states in the neocortexs. Nature 423:283-288. CrossRef Medline

Delord B, Klaassen A, Burnod Y, Guigon E (1996) An intrinsic bistable mechanism in neocortical pyramidal neurons might be involved in the generation of sustained discharge patterns related to working memory. Neural Network World 4:525-533.

Delord B, Klaassen AJ, Burnod Y, Costalat R, Guigon E (1997) Bistable behaviour in a neocortical neurone model. Neuroreport 8:1019-1023. CrossRef Medline

Dembrow NC, Chitwood RA, Johnston D (2010) Projection-specific neuromodulation of medial prefrontal cortex neurons. J Neurosci 30:1692216937. CrossRef Medline

Destexhe A, Paré D (1999) Impact of network activity on the integrative properties of neocortical pyramidal neurons in vivo. J Neurophysiol 81: 1531-1547. CrossRef Medline

Destexhe A, Rudolph M, Fellous JM, Sejnowski TJ (2001) Fluctuating synaptic conductances recreate in vivo-like activity in neocortical neurons. Neuroscience 107:13-24. CrossRef Medline

Egorov AV, Hamam BN, Fransén E, Hasselmo ME, Alonso AA (2002) Graded persistent activity in entorhinal cortex neurons. Nature 420:173178. CrossRef Medline

Faber ES, Sah P (2007) Functions of SK channels in central neurons. Clin Exp Pharmacol Physiol 34:1077-1083. CrossRef Medline

Fellous JM, Rudolph M, Destexhe A, Sejnowski TJ (2003) Synaptic background noise controls the input/output characteristics of single cells in an in vitro model of in vivo activity. Neuroscience 122:811-829. CrossRef Medline

Gee S, Ellwood I, Patel T, Luongo F, Deisseroth K, Sohal VS (2012) Synaptic activity unmasks dopamine $\mathrm{D}_{2}$ receptor modulation of a specific class of layer V pyramidal neurons in prefrontal cortex. J Neurosci 32:49594971. CrossRef Medline

Genet S, Delord B (2002) A biophysical model of nonlinear dynamics underlying plateau potentials and calcium spikes in Purkinje cell dendrites. J Neurophysiol 88:2430-2444. CrossRef Medline

Genet S, Sabarly L, Guigon E, Berry H, Delord B (2010) Dendritic signals command firing dynamics in a mathematical model of cerebellar Purkinje cells. Biophys J 99:427-436. CrossRef Medline 
Goldman MS, Levine JH, Major G, Tank DW, Seung HS (2003) Robust persistent neural activity in a model integrator with multiple hysteretic dendrites per neuron. Cereb Cortex 13:1185-1195. CrossRef Medline

Haj-Dahmane S, Andrade R (1997) Calcium-activated cation nonselective current contributes to the fast afterdepolarization in rat prefrontal cortex neurons. J Neurophysiol 78:1983-1989. CrossRef Medline

Helton TD, Xu W, Lipscombe D (2005) Neuronal L-type calcium channels open quickly and are inhibited slowly. J Neurosci 25:10247-10251. CrossRef Medline

Hounsgaard J, Kiehn O (1993) Calcium spikes and calcium plateaux evoked by differential polarization in dendrites of turtle motoneurones in vitro. J Physiol 468:245-259. CrossRef Medline

Kawasaki H, Palmieri C, Avoli M (1999) Muscarinic receptor activation induces depolarizing plateau potentials in bursting neurons of the rat subiculum. J Neurophysiol 82:2590-2601. CrossRef Medline

Koulakov AA, Raghavachari S, Kepecs A, Lisman JE (2002) Model for a robust neural integrator. Nat Neurosci 5:775-782. CrossRef Medline

Krnjević K, Pumain R, Renaud L (1971) The mechanism of excitation by acetylcholine in the cerebral cortex. J Physiol 215:247-268. CrossRef Medline

Larimer P, Strowbridge BW (2010) Representing information in cell assemblies: persistent activity mediated by semilunar granule cells. Nat Neurosci 13:213-222. CrossRef Medline

Lee RH, Heckman CJ (1998) Bistability in spinal motoneurons in vivo: systematic variations in persistent inward currents. J Neurophysiol 80:583593. CrossRef Medline

Loewenstein Y, Mahon S, Chadderton P, Kitamura K, Sompolinsky H, Yarom Y, Häusser M (2005) Bistability of cerebellar Purkinje cells modulated by sensory stimulation. Nat Neurosci 8:202-211. CrossRef Medline

MacLean JN, Watson BO, Aaron GB, Yuste R (2005) Internal dynamics determine the cortical response to thalamic stimulation. Neuron 48:811823. CrossRef Medline

Major G, Polsky A, Denk W, Schiller J, Tank DW (2008) Spatiotemporally graded NMDA spike/plateau potentials in basal dendrites of neocortical pyramidal neurons. J Neurophysiol 99:2584-2601. CrossRef Medline

Marder E, Calabrese RL (1996) Principles of rhythmic motor pattern generation. Physiol Rev 76:687-717. CrossRef Medline

McCormick DA, Shu Y, Hasenstaub A, Sanchez-Vives M, Badoual M, Bal T (2003) Persistent cortical activity: mechanisms of generation and effects on neuronal excitability. Cereb Cortex 13:1219-1231. CrossRef Medline

Miller P, Wang XJ (2006) Power-law neuronal fluctuations in a recurrent network model of parametric working memory. J Neurophysiol 95:10991114. CrossRef Medline

Milojkovic BA, Radojicic MS, Antic SD (2005) A strict correlation between dendritic and somatic plateau depolarizations in the rat prefrontal cortex pyramidal neurons. J Neurosci 25:3940-3951. CrossRef Medline

Naudé J, Paz JT, Berry H, Delord B (2012) A theory of rate coding control by intrinsic plasticity effects. PLoS Comput Biol 8:e1002349. CrossRef Medline

Ostojic S (2014) Two types of asynchronous activity in networks of excitatory and inhibitory spiking neurons. Nat Neurosci 17:594-600. CrossRef Medline

Perrier JF, Tresch MC (2005) Recruitment of motor neuronal persistent inward currents shapes withdrawal reflexes in the frog. J Physiol 562:507520. CrossRef Medline

Rekling JC, Feldman JL (1997) Calcium-dependent plateau potentials in rostral ambiguus neurons in the newborn mouse brain stem in vitro. J Neurophysiol 78:2483-2492. CrossRef Medline

Sanchez-Vives MV, McCormick DA (2000) Cellular and network mechanisms of rhythmic recurrent activity in neocortex. Nat Neurosci 3:10271034. CrossRef Medline

Schwindt PC, Spain WJ, Foehring RC, Chubb MC, Crill WE (1988) Slow conductances in neurons from cat sensorimotor cortex in vitro and their role in slow excitability changes slow conductances in neurons from cat sensorimotor cortex in vitro and their role in slow excitability changes. J Neurophysiol 59:450-467. CrossRef Medline

Seidemann E, Meilijson I, Abeles M, Bergman H, Vaadia E (1996) Simultaneously recorded single units in the frontal cortex go through sequences of discrete and stable states in monkeys performing a delayed localization task. J Neurosci 16:752-768. CrossRef Medline

Shafi M, Zhou Y, Quintana J, Chow C, Fuster J, Bodner M (2007) Variability in neuronal activity in primate cortex during working memory tasks. Neuroscience 146:1082-1108. CrossRef Medline

Shouval HZ, Gavornik JP (2011) A single spiking neuron that can represent interval timing: analysis, plasticity and multi-stability. J Comput Neurosci 30:489-499. CrossRef Medline

Sidiropoulou K, Lu FM, Fowler MA, Xiao R, Phillips C, Ozkan ED, Zhu MX, White FJ, Cooper DC (2009) Dopamine modulates an mGluR5mediated depolarization underlying prefrontal persistent activity. Nat Neurosci 12:190-199. CrossRef Medline

Silva LR, Amitai Y, Connors BW (1991) Intrinsic oscillations of neocortex generated by layer 5 pyramidal neurons. Science 251:432-435. CrossRef Medline

Tahvildari B, Fransén E, Alonso AA, Hasselmo ME (2007) Switching between "On" and "Off" states of persistent activity in lateral entorhinal layer III neurons. Hippocampus 17:257-263. CrossRef Medline

Thuault SJ, Malleret G, Constantinople CM, Nicholls R, Chen I, Zhu J, Panteleyev A, Vronskaya S, Nolan MF, Bruno R, Siegelbaum SA, Kandel ER (2013) Prefrontal cortex HCN1 channels enable intrinsic persistent neural firing and executive memory function. J Neurosci 33:1358313599. CrossRef Medline

Villalobos C, Shakkottai VG, Chandy KG, Michelhaugh SK, Andrade R (2004) SKCa channels mediate the medium but not the slow calciumactivated afterhyperpolarization in cortical neurons. J Neurosci 24:35373542. CrossRef Medline

Wang M, Ramos BP, Paspalas CD, Shu Y, Simen A, Duque A, Vijayraghavan S, Brennan A, Dudley A, Nou E, Mazer JA, McCormick DA, Arnsten AF (2007) 2A-adrenoceptors strengthen working memory networks by inhibiting cAMP-HCN channel signaling in prefrontal cortex. Cell 129: 397-410. CrossRef Medline

Wang XJ (2001) Synaptic reverberations underlying mnemonic persistent activity. Trends Neurosci 24:455-463. CrossRef Medline

Washburn DL, Anderson JW, Ferguson AV (2000) A subthreshold persistent sodium current mediates bursting in rat subfornical organ neurones. J Physiol 529:359-371. Medline

Yang CR, Seamans JK, Gorelova N (1996) Electrophysiological and morphological properties of layers V-VI principal pyramidal cells in rat prefrontal cortex in vitro. J Neurosci 16:1904-1921. CrossRef Medline

Zhang Z, Séguéla P (2010) Metabotropic induction of persistent activity in layers II / III of anterior cingulate cortex. Cereb Cortex 20:2948-2957. CrossRef Medline 